\title{
ON THE MULTIPLICITY OF AN INTEGRAL EXTENSION OF A LOCAL RING
}

\author{
DAVID G. WHITMAN
}

Abstract. The following theorem is proved: If $R$ is a local domain with field of quotients $F$ and $S$ is a local integral extension of $R$ contained in $F$, then the multiplicity of $R$ is greater than or equal to the multiplicity of $S$.

Throughout this paper, all rings considered will be commutative with an identity element. For necessary background and terminology, the reader is referred to [1].

It is well known (see [1]) that if $(R, M)$ is a local ring, then, for large $n$,

$$
l\left(R / M^{n+1}\right)=e_{0}\left(\begin{array}{c}
n+d \\
d
\end{array}\right)-e_{1}\left(\begin{array}{c}
n+d-1 \\
d-1
\end{array}\right)+\cdots+(-1)^{d} e_{d}
$$

where $d$ is the altitude of $R$. The integer $e_{0}$ is called the multiplicity of $R$, and is denoted $m(R)$. In [2, p. 207], Nagata shows that if $R$ is a local domain with field of quotients $F$ and $S$ is a finite local integral extension of $R$ contained in $F$, then $m(S) \leqq m(R)$. The purpose of this note is to indicate how the finiteness condition can be removed. In particular, the following is proved.

Theorem. Let $(R, M)$ be a local domain with field of quotients $F$ and let $(S, N)$ be a local integral extension of $R$ contained in $F$. Then $m(R) \geqq m(S)$.

Proof. Let $N=\left(a_{1}, \cdots, a_{n}\right)$, and let $T=R\left[a_{1}, \cdots, a_{n}\right]$. By the Hilbert Basis Theorem $T$ is Noetherian; also, $T$ is quasilocal since it is dominated by $S$ (see 10.7 of [1]). Thus $T$ is local and finite over $R$, and, by Nagata's result, it now follows that $m(R) \geqq m(T)$. So without loss of generality, it may be assumed that $M S=N$. This clearly implies that $M^{k} S=N^{k}$, for all $k$. Therefore $l\left(M^{k} / M^{k+1}\right) \geqq l\left(N^{k} / N^{k+1}\right)$ since these figures are equal to the minimum number of generators of $M^{k}$ and $N^{k}$ respectively. Now by summation, $l\left(R / M^{n}\right) \geqq l\left(S / N^{n}\right)$, for all $n$. Finally, by comparison of these two polynomials which have equal degree, it follows that $m(R) \geqq m(S)$. Q.E.D.

Received by the editors September 22, 1969.

AMS Subject Classifications. Primary 1395, 1320.

Key Words and Phrases. Local ring, integral extension, finite integral extension, Hilbert Basis Theorem, multiplicity. 
In concluslon, it should be remarked that Nagata [1, p. 205] has given an example of a local domain $R$ whose derived normal ring $R^{\prime}$ is local but not finite over $R$.

\section{BIBLIOGRAPHY}

1. M. Nagata, Local rings, Interscience Tracts in Pure and Appl. Math., no. 13, Interscience, New York, 1962. MR 27 \#5790.

2. - The theory of multiplicity in general local rings, Internat. Sympos. Algebraic Number Theory (Tokyo \& Nikko, 1955), Science Council of Japan, Tokyo, 1956, pp. 191-226. MR 18, 637.

San Diego State College, San Diego, California 92115 